OPEN ACCESS

Edited by: Qingfeng Chen, Institute of Molecular and Cell Biology (A*STAR), Singapore

Reviewed by:

Amy L. MacNeill, Colorado State University, United States Johan Van Weyenbergh, KU Leuven, Belgium

*Correspondence: Liang Cheng liangcheng@whu.edu.cn Lishan Su

Isu@ihv.umaryland.edu

Specialty section: This article was submitted to Viral Immunology, a section of the journal

Frontiers in Immunology

Received: 25 February 2021 Accepted: 27 April 2021 Published: 18 May 2021

Citation:

Cheng L, Li G, Pellegry CM, Yasui F, Li F, Zurawski SM, Zurawski G, Levy Y, Ting JP-Y and Su L (2021) TLR9- and CD40-targeting Vaccination Promotes

Human B Cell Maturation and IgG Induction via $p D C$-Dependent Mechanisms in Humanized Mice.

Front. Immunol. 12:672143. doi: 10.3389/fimmu.2021.672143

\section{TLR9- and CD40-Targeting Vaccination Promotes Human B Cell Maturation and IgG Induction via pDC-Dependent Mechanisms in Humanized Mice}

\author{
Liang Cheng ${ }^{1,2 *}$, Guangming $L^{1,3}$, Caroline Marnata Pellegry ${ }^{1}$, Fumihiko Yasui ${ }^{1,4}$, \\ Feng $L_{i}{ }^{1,5}$, Sandra M. Zurawski ${ }^{6}$, Gerard Zurawski ${ }^{6}$, Yves Levy ${ }^{7,8}$, Jenny P.-Y. Ting ${ }^{1,9,10}$ \\ and Lishan $\mathrm{Su}^{1,3^{*}}$

\begin{abstract}
1 Lineberger Comprehensive Cancer Center, University of North Carolina at Chapel Hill, Chapel Hill, NC, United States, 2 Frontier Science Center for Immunology and Metabolism, Medical Research Institute, Wuhan University, Wuhan, China, ${ }^{3}$ Division of Virology, Pathogenesis and Cancer, Institute of Human Virology, Department of Pharmacology, University of Metropolitan Institute of Medical Science, Tokyo, Japan, ${ }^{5}$ Guangzhou Eighth People's Hospital, Guangzhou Medical University, Guangzhou, China, ${ }^{6}$ Baylor Institute for Immunology Research, Vaccine Research Institute, INSERM U955, Dallas, Clinique, Créteil, France, ${ }^{8}$ Vaccine Research Institute, Université Paris-Est Créteil, Faculté de Médecine, INSERM U955, Créteil, France, ${ }^{9}$ Department of Genetics, University of North Carolina at Chapel Hill, Chapel Hill, NC, United States,

${ }^{10}$ Department of Microbiology-Immunology, University of North Carolina at Chapel Hill, Chapel Hill, NC, United States
\end{abstract} \\ Maryland School of Medicine, Baltimore, MD, United States, ${ }^{4}$ Department of Microbiology and Cell Biology, Tokyo \\ TX, United States, ${ }^{7}$ Assistance Publique-Hôpitaux de Paris, Groupe Henri-Mondor Albert-Chenevier, Service Immunologie
}

Mice reconstituted with a human immune system (humanized mice) provide a robust model to study human immunology, vaccinology, and human infectious diseases. However, the development and function of B cells in humanized mice is impaired. B cells from humanized mice are immature and are impaired in lgM to lgG isotype switch in response to infection or vaccination. In the present study we report that Toll-like receptor 9 (TLR9) agonist CpG-B combined with CD40-targeting vaccination triggered human $\mathrm{B}$ cell immunoglobin class-switch from $\operatorname{lgM}^{+}$to $\operatorname{lgG}^{+} B$ cells in humanized mice. Human $B$ cells from mice vaccinated with $\mathrm{CpG}-\mathrm{B}$ as adjuvant were more mature in phenotype and produced significant levels of both total lgG and antigen-specific lgG. We found that CpG$\mathrm{B}$ treatment activated human pDCs (plasmacytoid dendritic cells) in vivo to induce interferon-alpha (IFN- $\alpha$ )expression in humanized mice. Pre-depletion of human pDC in vivo abrogated the adjuvant effect of CpG-B. Our results indicate that TLR9 and CD40targeting vaccination triggers human $B$ cell maturation and immunoglobulin class-switch in a pDC-dependent manner in humanized mice. The findings also shed light on induction of human IgG antibodies in humanized mouse models.

Keywords: plasmacytoid dendritic cell, IFN-alpha, immunoglobin class-switch, IgG induction, CD40-targeting vaccination, B cell maturation, CpG-B 


\section{INTRODUCTION}

Recent development of humanized mice provides robust models to study infection, pathogenesis, and therapy of human viruses $(1,2)$. We and others have shown before that a functional human immune system was developed in immunodeficient mice after adoptive transfer of human hematopoietic stem cells (HSCs) (38). Major human immune subtype such as $\mathrm{pDC}$, mDC, monocyte, $\mathrm{T}$ and $\mathrm{B}$ cells can be detected in peripheral blood and lymphoid organs 3 months after HSCs transfer (7). Humanized mice can initiate innate immunity and antigenspecific $\mathrm{T}$ cell response to vaccine or infection (9-12). However, although B cell are developed in humanized mice, those cells are immature (13). B cells from humanized mice shows $\mathrm{CD} 24^{\mathrm{int} / \mathrm{hi}} \mathrm{CD} 38^{\mathrm{hi}}$ immature phenotype and express high levels of CD10, another immature B cell marker (14). Moreover, $B$ cells from humanized mice are predominately $\mathrm{IgM}^{+}$with few $\mathrm{IgG}^{+} \mathrm{B}$ cells (15). The cells are impaired in IgM to IgG isotype switch in response to infection or vaccination $(14,15)$.

Toll like receptor (TLRs) are expressed by various immune cells such as plasmacytoid dendritic cells (pDCs), monocytes and myeloid dendritic cells (mDCs) and B cells $(16,17)$. They can sense the microbial components named pathogen-associated molecular patterns (PAMPs) $(16,17)$. Stimulation of TLR signaling by synthetic or natural TLR ligands (TLR-Ls) results in up-regulation of MHC class II molecules, co-stimulatory molecules, and cytokines in different kind of cells in the immune system, especially innate immune cells $(18,19)$. These synthetic or natural agonists for TLRs are potential new vaccine adjuvants $(20,21)$. We and others have shown before that TLRLs can efficiently activate human innate immune cells from humanized mice both in vitro and in vivo $(9,11)$. Importantly, we proved that CpG-B, R848 and Poly I:C can enhance antigenspecific $\mathrm{T}$ cells response to a CD40-targeting HIV vaccine in humanized mice (9). Moreover, we recently reported that therapeutic treatment with a CD40-targeting HIV vaccine plus poly I:C as adjuvant can significantly reduce HIV-1 reservoir in HIV-infected humanized mice (12). These results indicated that humanized mice serve as a relevant model to develop and evaluate novel vaccines and adjuvants to human infectious disease.

As B cells from humanized mice fails to efficiently transition from $\mathrm{IgM}^{+}$to $\mathrm{IgG}^{+} \mathrm{B}$ cells, it is still questionable to use humanized mouse model to evaluate humoral immune response to vaccines (13). Efforts have been made to improve B cell IgM to IgG class-switch and antigen-specific antibody production in humanized mice in recent years. It was reported that GM-CSF and IL-4 stimulate humoral responses in humanized mice by promoting dendritic cell, $\mathrm{T}$ and $\mathrm{B}$ cell maturation (22). Transgenes expressing human stem cell factor, granulocyte-macrophage colony stimulating factor and interleukin-3 was reported to improve B cell development in humanized mice (23). The expression of human IL- 6 by knocking-in human IL-6 gene to its respective mouse locus also increased class switched memory B cells and serum immunoglobulin G (IgG) after HSCs reconstitution (24). Stimulation of TLR signaling by synthetic or natural TLR ligands (TLR-Ls) results in cytokines induction, as well as upregulation of MHC class II molecules and co-stimulatory molecules in innate immune cells $(9,11)$. Synthetic CpG oligodeoxynucleotides ( $\mathrm{CpG}$ ODNs), which signal through TLR9, are approved by FDA (DYNAVAX) as HBV vaccine adjuvants after human clinical trials. The principal TLR9expressing cells in humans are plasmacytoid DCs (pDCs) and B cells $(25,26)$. We speculated that targeting TLR9 would serve as a good stratagem to improve humoral immune response to vaccines in humanized mice. However, knowledge about how CpG ODNs activate pDC and enhance B cell response in vivo in humans is still limited.

In the present study we tested whether targeting TLR9 by CpG-B can overcome the deficiency of B cell response in humanized mice. We found that $\mathrm{CpG}-\mathrm{B}$ combined with $\mathrm{CD} 40$ targeting vaccination enhanced $\mathrm{B}$ cell maturation, triggered human $\mathrm{B}$ cell immunoglobin class-switch from $\operatorname{IgM}^{+}$to $\operatorname{IgG}^{+} \mathrm{B}$ cells, and induced antigen-specific IgG response. Furthermore, we found that pDCs were essential for the adjuvant activity of CpG-B. Our results indicate that TLR9 and CD40-targeting vaccination triggers human $\mathrm{B}$ cell maturation, IgM to IgG immunoglobulin class-switch in a pDC-dependent manner in humanized mice.

\section{MATERIALS AND METHODS}

\section{Construction of Humanized Mice}

We constructed humanized NRG (NOD-Rag2 ${ }^{-/-} \gamma_{C}^{-/-}$) mice by reconstitution with human fetal liver (17 to 22 weeks of gestational age) derived $\mathrm{CD}_{3} 4^{+}$hematopoietic progenitor cells (Advanced Bioscience Resources, Alameda, CA) as previously reported (27). Briefly, CD $34^{+}$hematopoietic progenitor cells purified from fetal liver were injected into the liver of newborn (1-3 day) NRG mice. Human immune reconstitution was detected by flow cytometry 12 weeks after transplantation. All animal studies were approved by the University of North Carolina Institutional Animal Care and Use Committee (IACUC).

\section{Ethics Statement}

Human fetal liver was obtained from elective or medically indicated termination of pregnancy through a non-profit intermediary working with outpatient clinics (Advanced Bioscience Resources, Alameda, CA). Informed consent of the maternal donor is obtained in all cases, under regulation governing the clinic. The use of the tissue in the research had no influence on the decision regarding termination of the pregnancy. The project was reviewed by the University's Office of Human Research Ethics, which has determined that this submission does not constitute human subjects research as defined under federal regulations [45 CFR 46.102 ( $\mathrm{d}$ or f) and 21 CFR 56.102(c)(e)(l)] and does not require IRB approval. All animal experiments were conducted following NIH guidelines for housing and care of laboratory animals and in accordance with The University of North Carolina at Chapel Hill with protocols approved by the institution's Institutional Animal Care and Use Committee (IACUC ID: 14-100). 


\section{Vaccination}

Recombinant anti-human CD40 antibody fused to 5 HIV-1 long peptide regions ( $\alpha \mathrm{CD} 40$-HIV5pep) was produced as previously reported (28), except that the flexible linker and HIV peptide sequences were reconfigured and the variable regions CD40 antibody were changed to a human framework. Humanized mice were intramuscularly (half dose) and intraperitoneally (half dose) injected with $10 \mu \mathrm{g} \alpha \mathrm{CD} 40$ HIV5pep or recombinant hemagglutinin protein alone or with $50 \mu \mathrm{g}$ of CpG-B at week0, week3 and week6. Splenocytes from vaccinated humanized mice were collected 7 days after the third vaccination.

\section{Detection of Cytokines}

Human IFN- $\alpha$ was detected by enzyme-linked immunosorbent assay using the human IFN- $\alpha$ pan ELISA kits purchased from Mabtech. A high sensitivity immunology multiplex assay (Luminex) (Millipore, Billerica, Massachusetts, USA) was used to measure human IL-6 in plasma of humanized mice according to the manufacturer's instructions.

\section{Flow Cytometry}

Single cell suspensions prepared from peripheral blood, spleen of humanized mice was stained with surface markers and analyzed on a CyAn ADP (Dako). FITC-conjugated anti-human CD40, CD24, PE-conjugated anti-human CD303, CD38, PE/Cy5conjugated anti-human CD86, IgG, PE/Cy7-conjugated antihuman HLA-DR, PB-conjugated anti-human CD4, IgM, APCconjugated anti-human CD10 and APC/Cy7-conjugated antihuman CD45 were purchased from Biolegend. Pacific orangeconjugated anti-mouse CD45, PE/Texas red-conjugated antihuman CD19 and LIVE/DEAD Fixable Aqua (LD7) Dead Cell Stain Kit were purchased from Invitrogen. Data were analyzed using Summit4.3 software (Dako).

\section{Total IgM and IgG Detection}

Total IgM and IgG were detected by ELISA kits, purchased from Bethyl Laboratories,int.(Cat. No. E80-104 and Cat. No. E80-100) according to the protocols.

\section{Detection of Total and Antigen-Specific IgG-Secreting Cells by ELISpot}

IgG-secreting cells were detected by using ELISpot ${ }^{\text {PLUS }}$ for Human IgG kits (Product Code: 3850-2HW-Plus) according to the protocols. In brief, 96-well ELISpot plates were coated with an IgG capture antibody (for total IgG detection) or antigen $(\alpha C D 40-H I V 5 p e p$, for specific IgG detection) in PBS overnight at $4^{\circ} \mathrm{C}$. Then the plate was blocked with $200 \mu \mathrm{l} /$ well of medium containing $10 \%$ FBS for at least 30 minutes at room temperature. The pre-activated splenocytes (splenocyte from humanized mice cultured ex vivo in the present of R848 $(1 \mu \mathrm{g} / \mathrm{ml})$ and IL-2 $(10 \mathrm{u} /$ $\mathrm{ml}$ ) for 48hours) were then added to the ELISpot plate and incubated in a $37^{\circ} \mathrm{C}$ incubator with $5 \% \mathrm{CO}_{2}$ for $16-24$ hours. Plates were washed 5 times with PBS and incubated with IgG detection mAbs for 2 hours at room temperature. Then, 1:1,000 dilution of streptavidin-HRP was added and incubated for 1 hour at room temperature. Followed by washing 5 times, the plates were developed with TMB substrate solution until distinct spots emerge. The spots were inspected and counted by using an ELISpot reader.

\section{Antigen-Specific IgG Detection in Serum}

96-well ELISA plates were coated with antigen $(\alpha C D 40$ HIV5pep, $10 \mu \mathrm{g} / \mathrm{ml}$ or HA protein, $10 \mu \mathrm{g} / \mathrm{ml}$ ) in PBS overnight at $4^{\circ} \mathrm{C}$. Then the plate was blocked with $200 \mu \mathrm{l} /$ well of medium containing $10 \%$ FBS for at least 30 minutes at room temperature. Then $50 \mu \mathrm{l}$ of serum from vaccinated mice was added into the plate and incubated for 2 hours. Plates were washed 5 times with PBS and incubated with IgG detection mAbs for 2 hours at room temperature. Then, 1:1,000 dilution of streptavidin-HRP was added and incubated for 1 hour at room temperature. Followed by washing 5 times, the plates were developed with TMB substrate solution. The reaction was stopped with ELISA Stop Solution (Cat. No. E115), and the plate was read at $450 \mathrm{~nm}$.

\section{pDC Depletion In Vivo}

A $\mathrm{mAB}$ specific to $\mathrm{BDCA} 2$, clone $15 \mathrm{~B}$, was used to deplete $\mathrm{pDCs}$ in humanized mice through i.p. injection $(4 \mathrm{mg} / \mathrm{kg})$. In brief, 15B was applied to mice at 3 and 1 day before each vaccination. At day 0 , the mice were either treated with $\mathrm{CpG}-\mathrm{B}$ or received vaccination treatment.

\section{Statistical Analysis}

In all experiments, significance levels of data were determined by using Prism5 (GraphPad Software). Experiments were analyzed by two-tailed Student's t-test, one-way analysis of variance (ANOVA) and Tukey's multiple comparisons test, or Spearman rank correlation test as indicated in figure legends. A p value less than 0.05 was considered significant. The number of animals was specified in each figure legend.

\section{RESULTS}

\section{TLR9 and CD40-Targeting Vaccination Promotes IgG Induction in Humanized Mice}

Human B cell development and function are compromised in humanized mice, and B cells are impaired to undergo immunoglobin class switch from IgM to IgG. We tested whether targeting TLR-9 by CpG-B combined with a CD40-targeting vaccine can enhance $B$ cell immunity in humanized mice. Humanized mice were immunized with $\alpha \mathrm{CD} 40$-HIV5pep protein with or without CpG-B. B cell responses were evaluated one week after the boost vaccination. As reported (15), we found that B cells from PBMCs and spleen of PBS-treated humanized mice did not express surface IgG (Figures 1A, B). Vaccination with $\alpha C D 40$ HIV5pep protein alone failed to induce IgG expression on B cells (Figures 1A, B). Impressively, we found that, in humanized mice vaccinated with $\mathrm{CpG}-\mathrm{B}$ together with $\alpha \mathrm{CD} 40-\mathrm{HIV} 5$ pep protein, around $12 \%$ (7.0\% to $21.3 \%)$ of B cells from PBMCs and 5\% (3.2\% to $6.3 \%$ ) of $\mathrm{B}$ cells from the spleens of humanized mice were $\operatorname{IgG}^{+} \mathrm{B}$ 
cells (Figures 1A, B). CpG-B together with $\alpha$ CD40-HIV5pep vaccination also increased total human IgM level by-2 fold (Figure 1C). Human IgG, which is rarely detectable in humanized mice, reached $100 \mu \mathrm{g} / \mathrm{ml}$ in $75 \%$ (3/4) humanized mice after $\mathrm{CpG}-\mathrm{B}$ together with CD40-targeting vaccination (Figure 1C). We also detected by ELISpot assay around 1,500 cells per million B cells from spleens of $75 \%$ (3/4) humanized mice received $\mathrm{CpG}-\mathrm{B}$ plus $\mathrm{CD} 40$-targeting vaccination that were producing IgG, while no IgG-producing cells were detected in control groups (Figure 1D).

We next tested whether antigen-specific IgG was induced by CD40-targeting vaccination together with CpG-B. We found that 75\% (3/4) mice from CpG-B/CD40-targeting vaccine group produced specific IgG, while no antigen specific IgG was detectable in control groups (Figure 1E). Expression of activation-induced cytidine deaminase (AID) by germinal center (GC) B cells is important for class switch and recombination (29). We found that AID expression was significantly increased ( $>3$-fold) in splenocytes from CpG-B plus CD40-targeting vaccination mice than cells from control groups (Figure 1F).

Together, our data indicate that TLR9 agonist CPG-B with a CD40-targeting vaccine induces immunoglobulin class-switch and enhances Ag-specific IgG responses in 75\% (3/4) humanized mice tested.

\section{CpG-B Promotes Maturation and Activation of B Cells in Humanized Mice}

We next detected the phenotype of human B cells after vaccination. As reported (14), we found that most B cells from control mice were $\mathrm{CD} 24^{\text {high }} \mathrm{CD} 38^{\text {high }}$ which indicated immature phenotype (Figures 2A, B). CD40-targeting vaccination alone

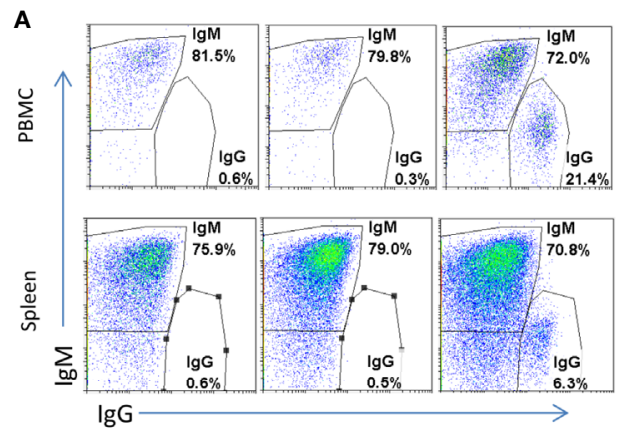

B
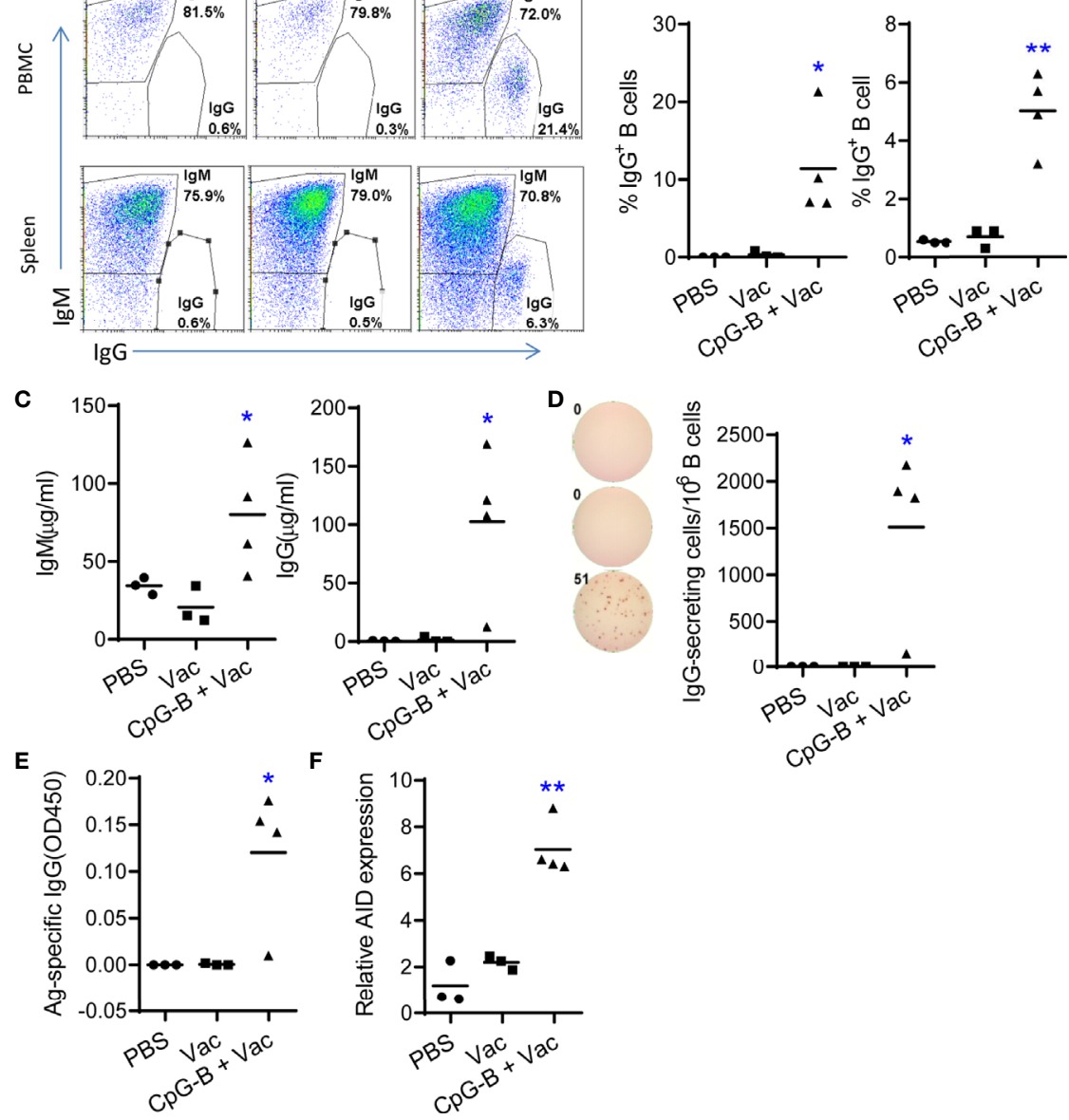

FIGURE 1 | TLR9 agonist CpG-B promotes lgG responses in humanized mice vaccinated with CD40-targeting vaccine. Humanized mice were treated with PBS control $(n=3)$ or vaccinated with $\alpha$ CD40-HIV5pep $(n=3)$ alone or vaccinated with $\alpha$ CD40-HIV5pep plus CpG-B ( $n=4)$ at week0, week3 and week6. At week 7, mice were sacrificed. (A, B) The expression of IgM and IgG on B cells from PBMCs and spleens was detected by FACS. (C) The total level of IgM and IgG in the plasma was detected by ELISA. (D) Splenocyte from humanized mice were cultured ex vivo in the present of R848(1 $\mathrm{gg} / \mathrm{ml})$ and IL-2(10 $\mathrm{u} / \mathrm{ml})$ for $48 \mathrm{hours}$, the cells were used for total IgG detection by ELISpot. (E) Antigen specific IgG level in the plasma was detected by ELISA. (F) The expression of activation-induced cytidine deaminase (AID) in spleen cells was detected by RT-PCR. Each dot represents one individual mouse, bars indicate mean. ${ }^{*} \mathrm{P}<0.05$, ${ }^{* *} \mathrm{P}<0.01$, by unpaired, twotailed Student's t-test comparing the two vaccinated groups. 
did not change the percentage of $\mathrm{CD} 38^{\text {high }} \mathrm{CD} 24^{\text {high }}$ immature $\mathrm{B}$ cells. CpG-B together with CD40-targeting vaccination decreased the percentage of $\mathrm{CD} 38^{\text {high }} \mathrm{CD} 24^{\text {high }} \mathrm{B}$ cells and increased the percentage of mature $B$ cells with
$\mathrm{CD} 38^{\text {int }} \mathrm{CD} 24^{\text {int }}$ or $\mathrm{CD} 38^{\text {low }} \mathrm{CD} 24^{\text {int }}$ phenotype (Figures 2A, B). We also find that CpG-B together with CD40-targeting vaccination increased the percentage of $\mathrm{CD}^{-} 0^{-} \mathrm{B}$ cells (Figures 2C, D). In addition, CpG-B together with CD40-targeting

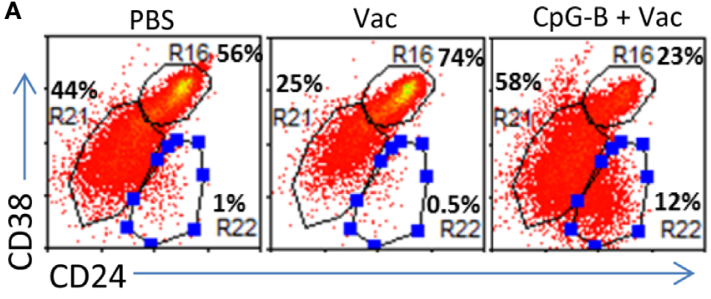

B

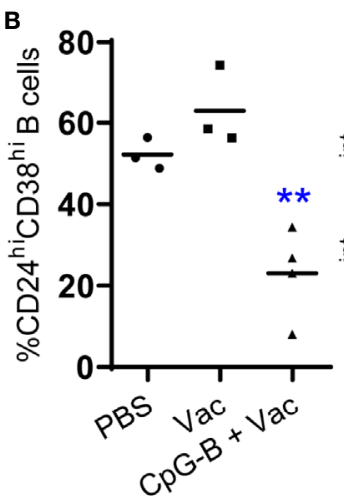

C

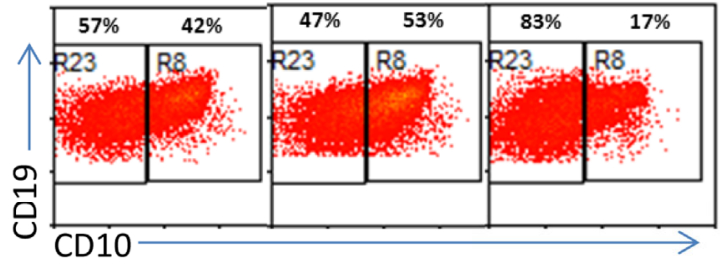

$\mathbf{E}$
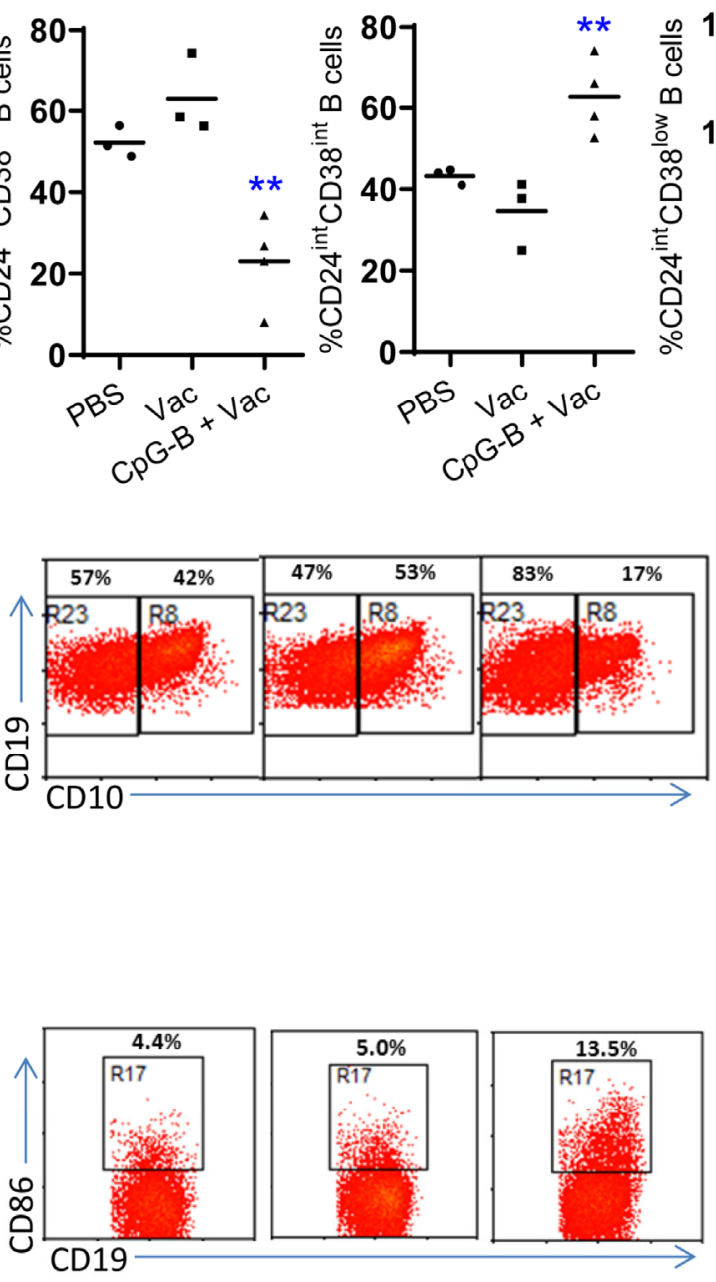

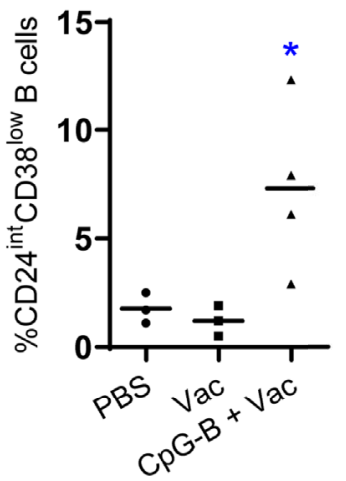
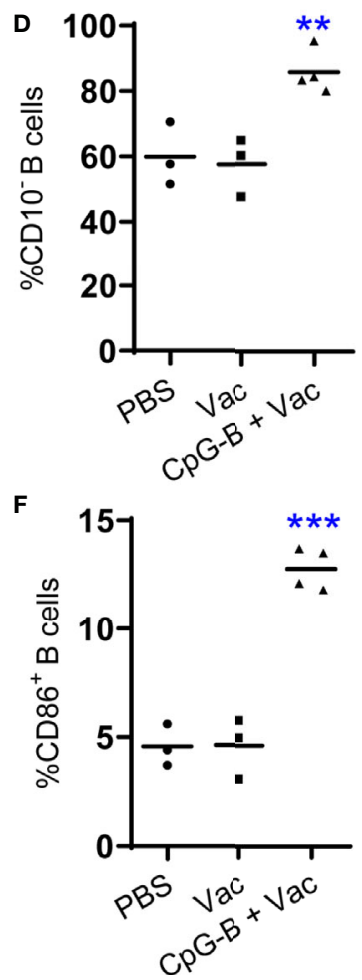

FIGURE 2 | CpG-B promotes maturation and activation of B cells in humanized mice. Humanized mice were vaccinated as in Figure 1. The phenotype of human B cells $\left(\mathrm{hCD} 45^{+} \mathrm{CD} 19^{+}\right)$from spleens of mice was detected by FACS. Representative dot plot $(\mathbf{A}, \mathbf{C}, \mathbf{E})$ and Summarized data $(\mathbf{B}, \mathbf{D}, \mathbf{F})$ showing the expression of CD38and CD24, CD10 and CD86 on human B cells. Each dot represents one individual mouse with n=3 in PBS group, $n=4$ in Vac group and n=4 in CpG-B plus Vac group. Bars indicate mean. ${ }^{\star} \mathrm{P}<0.05,{ }^{\star \star} \mathrm{P}<0.01,{ }^{\star \star \star} \mathrm{P}<0.001$, by unpaired, two-tailed Student's t-test comparing the two vaccinated groups. 
vaccination induced $\mathrm{CD}^{+} 6^{+}$activated $\mathrm{B}$ cells (Figures 2E, F). These data indicated that $\mathrm{CpG}-\mathrm{B}$ plus CD40-targeting vaccination induce $B$ cells maturation and activation.

\section{CpG-B Induces IFN- $\alpha$ Production in pDC Dependent Manner in Humanized Mice}

Dendritic cells are key to initiate and control immune responses. Plasmacytoid dendritic cells (pDC) represent a unique dendritic cell subtype and are specialized to produce large amount of IFN$\alpha$ upon viral infection (30). It was reported that $\mathrm{pDCs}$, through IFN- $\alpha$ and IL-6, are critical for the induction of IgG from human blood mononuclear cells in response to influenza virus
(31). We found that CpG-B treatment activated human pDCs (Figure 3A) and induced IFN- $\alpha$ and IL-6 production in humanized mice (Figure 3B). TLR9, the receptor for CpG-B, is preferentially expressed by $\mathrm{pDC}$ and $\mathrm{B}$ cells of human immune system. To investigate whether $\mathrm{pDC}$ contributed to IFN- $\alpha$ production after CpG-B treatment, we used a monoclonal antibody which can efficiently and specifically deplete pDC in vivo in humanized mice (32) (Figure 3C). We found that predepletion of $\mathrm{pDC}$ abrogated IFN- $\alpha$ production after CpG-B treatment (Figure 3D). The results indicate that $\mathrm{CpG}-\mathrm{B}$ treatment in humanized mice induces IFN- $\alpha$ expression thought a pDC dependent manner.

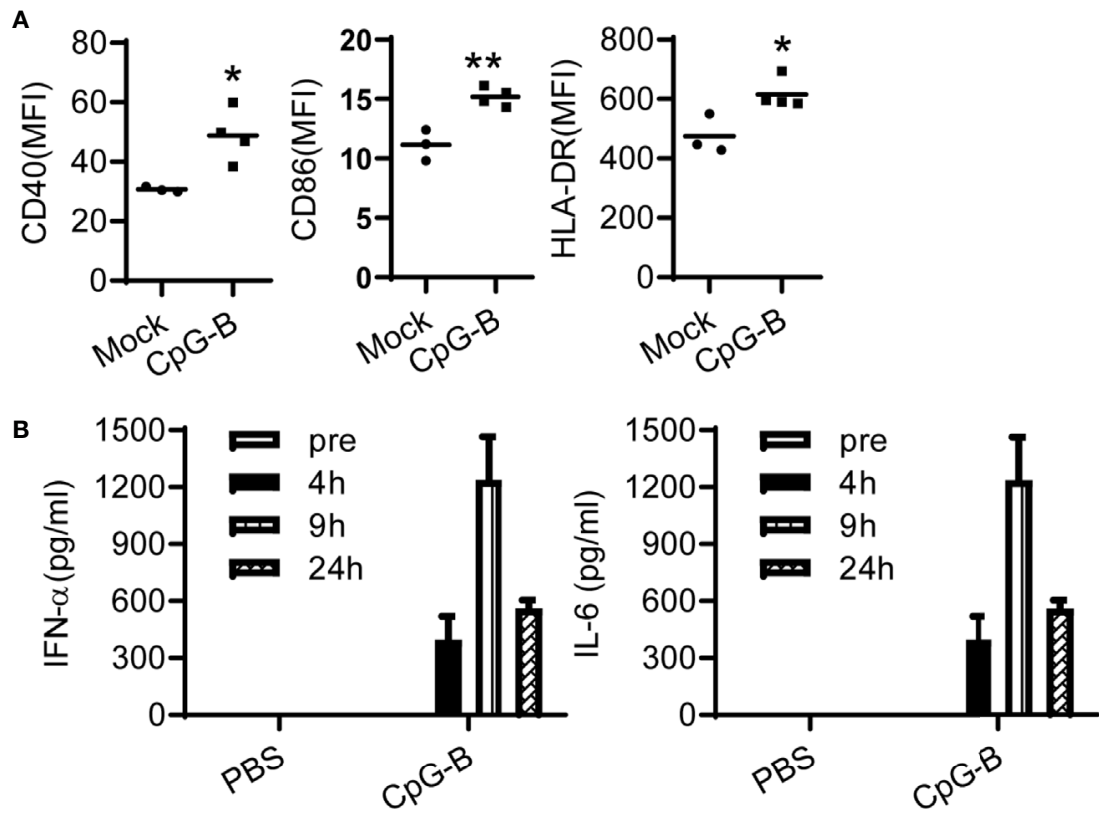

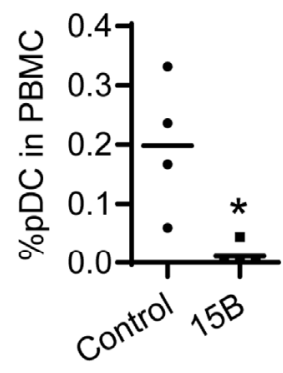

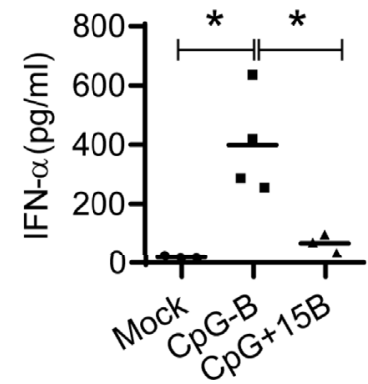

FIGURE 3 | CpG-induced IFN-a production in vivo is dependent on human pDCs in humanized mice. (A, B) Humanized mice were treated with PBS (n=3) or CpGB (50ug/mouse, i.p., n=4). (A) IFN- $\alpha$ and IL-6 level in plasma at indicated timepoint post treatment were detected by ELISA. (B) The expression of CD40, CD86 and HLA-DR on pDC $\left(\mathrm{CD}^{+}{ }^{+} \mathrm{CD}_{303^{+}}\right)$from spleens at 24 hours post-treatment was detected by FACS. (C) Humanized mice were pretreated with either isotype control $(n=3)$ or pDC depletion monoclonal antibody (clone 15B, $200 \mu \mathrm{g} /$ mouse, i.p., $n=4$ ) at day -3 and -1 . The percentage of pDC in human CD45+ cells from blood were detected by FACS. (D) Humanized mice were pretreated with either isotype control $(n=4)$ or pDC depletion monocolonal antibody (clone 15B, $200 \mu \mathrm{g} / \mathrm{mouse}$, i.p., $\mathrm{n}=3$ ) at day -3 and -1 . At day0, mice were treated PBS (Mock, $n=4)$ with CpG-B $(n=4)$. IFN-a level in plasma was detected 24 hours after CpG-B treatment. Each dot represents one individual mouse, bars indicate mean. ${ }^{*} P<0.05$, ${ }^{\star *} P<0.01$, by unpaired, two-tailed Student's t-test (A, C) or by one-way analysis of variance (ANOVA) and Tukey's multiple comparisons test (D). 


\section{TLR9 and CD40-Targeting Vaccination Depends on pDC to Promote Human B Cell Maturation and IgG Induction in Humanized Mice}

We next investigated whether CpG-B and CD40-targeting vaccination induced $\mathrm{B}$ cell maturation and IgG production in vivo dependent on $\mathrm{pDC}$. We vaccinated humanized mice with CpG-B and CD40-targeting vaccine in the presence or absence of $\mathrm{pDC}$ and then detected $\mathrm{B}$ cell phenotype and antibody response. As expected, pre-depletion of pDC by monoclonal antibody abrogated $\mathrm{CpG}-\mathrm{B}$ induced IFN- $\alpha$ production (Figure 4A). Interestingly, $\mathrm{CpG}-\mathrm{B}$ and $\mathrm{CD} 40$-targeting vaccine induced $\mathrm{B}$ cell maturation was also abrogated in the absence of $\mathrm{pDC}$ (Figure 4B). In the absence of $\mathrm{pDC}, \mathrm{CpG}-\mathrm{B}$ and CD40targeting vaccination also failed to induce total IgG (Figure 4C) and antigen-specific IgG (Figure 4D). Furthermore, we found that the IFN- $\alpha$ level in plasma of mice correlated with total and specific IgG production in vaccinated hu-mice (Figures 4E, F).

\section{CD40-Targeting Is Important for the Vaccine to Induce Class-Switch and IgG Production}

CD40 is a co-stimulatory receptor expressed by a range of APCs, including DCs (33). Thus, targeting CD40 offers the potential advantage of inducing DC maturation and delivery of antigen to CD40 induced antigen-specific humoral and cellular immune response $(34,35)$. We next determined whether $\mathrm{CD} 40$-targeting is important to induce class-switch and IgG production in humanized mice. Humanized mice were immunized with recombinant hemagglutinin protein (HA) with or without CpG-B. HA protein alone failed to induce specific IgG (Figure 5). HA with CpG-B as adjuvant, although induce IFN- $\alpha$ production and CD86 expression on $\mathrm{pDC}$, also did not induce specific IgG response to HA protein (Figure 5). These results indicate that CD40-targeting is important for the vaccine to induce class-switch and IgG production.

Taken together, we conclude that TLR9- and CD40-targeting vaccination promotes human $B$ cell maturation and $\mathrm{IgG}$ response via pDCs dependent mechanisms in humanized mice.
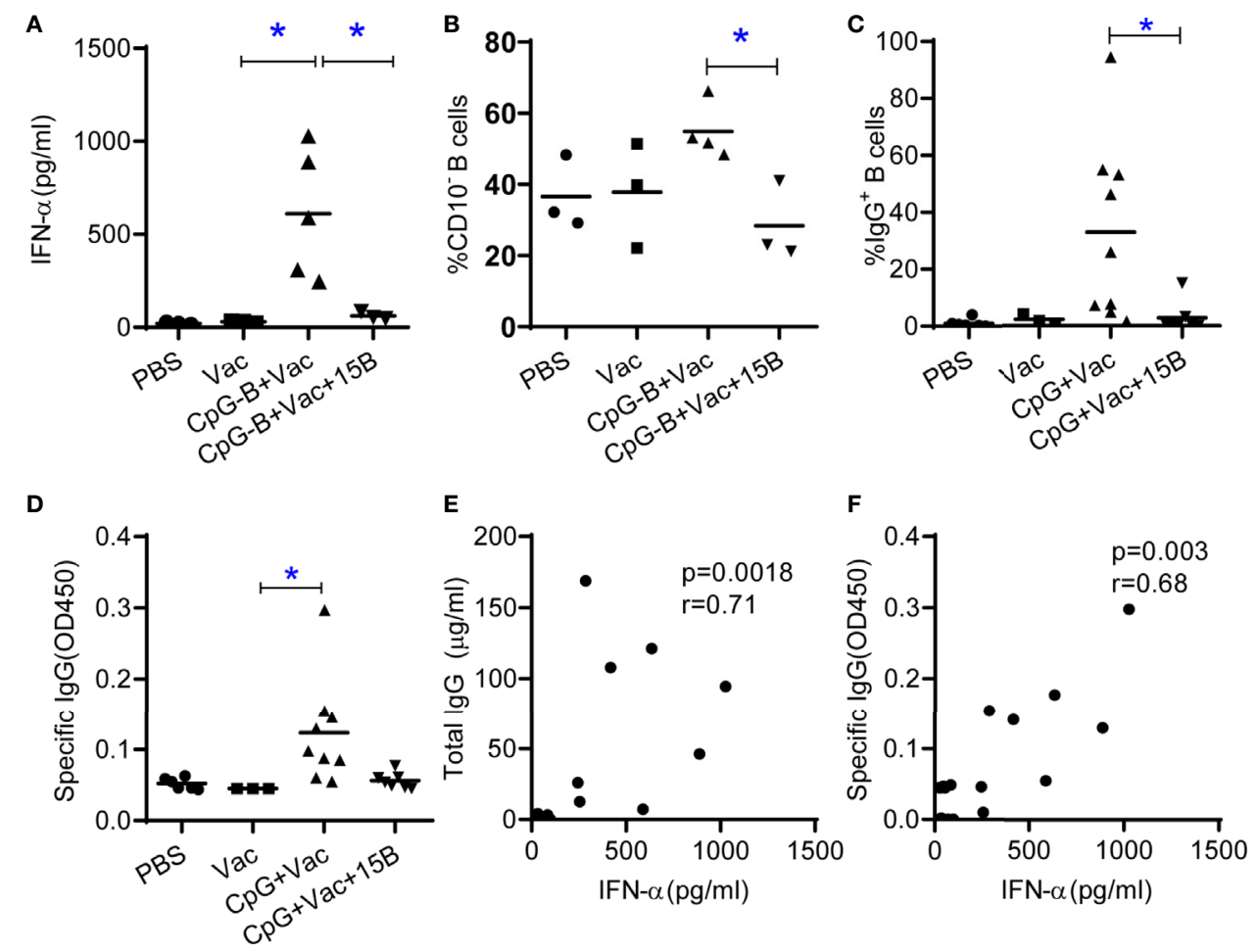

FIGURE 4 | TLR9 and CD40-targeting vaccination depends on pDC to promote human B cell maturation and IgG induction in humanized mice. Humanized mice were vaccinated as in Figure1 except one group of the mice were treated with pDC depletion Ab before vaccination (A) IFN- $\alpha$ in the plasma 24 hours after vaccination. (B) Expression of CD10 on B cells from spleen at termination. (C) Total IgG level in serum was detected by ELISA. (D) Antigen specific IgG level in the plasma was detected by ELISA. Each dot represents one individual mouse, bars indicate mean. Shown are representative data (PBS, $n=3$; Vac, $n=3 ; \mathrm{CpG}-\mathrm{B}+\mathrm{Vac}$, $n=4 ; C p G+V a c+15 B, n=3$, for $A$ and $B$ ) or combined data (PBS, $n=6$; Vac, $n=3$; CpG-B+Vac, $n=9$; CpG+Vac+15B, $n=7$, for $C$ and $D$ ) of two independent experiments with mean values. ${ }^{*} P<0.05$, by one-way analysis of variance (ANOVA) and Tukey's multiple comparisons test. (E, $\mathbf{F}$ ) Correlation analysis between the IFN- $\alpha$ levels in plasma and total IgG (E) and specific lgG (F) levels in plasma (Spearman rank correlation test). $r$, correlation coefficient. 


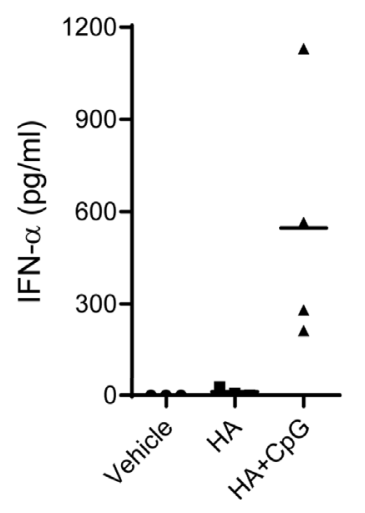

B

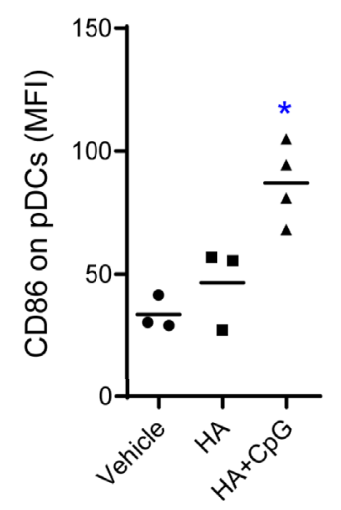

C

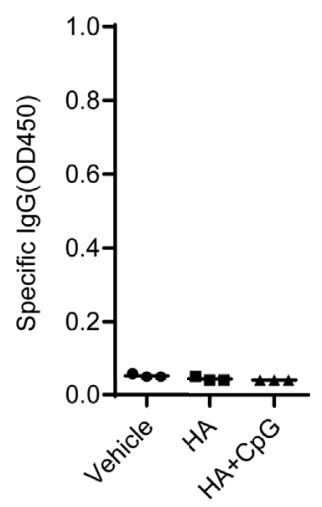

FIGURE 5 | CD40-targeting vaccination is required to induce lgG-response in humanized mice. Humanized mice were treated with PBS control (Vehicle, n=3) or vaccinated with recombinant hemagglutinin protein ( $\mathrm{HA}, \mathrm{n}=3,10 \mu \mathrm{g} /$ mouse) alone or vaccinated with $\mathrm{HA}$ plus $\mathrm{CpG}-\mathrm{B}$ ( $\mathrm{n}=4$ ) at week0, week3 and week6. (A) IFN-a levels in plasma in plasma at 24 hours post first vaccination was detected by ELISA. (B) The expression of CD86 on pDC (CD4 $\left.{ }^{+} \mathrm{CD} 303^{+}\right)$from PBMC at 24 hours post-treatment was detected by FACS. (C) Antigen specific IgG level in the plasma was detected by ELISA. Each dot represents one individual mouse, bars indicate mean. ${ }^{*} \mathrm{P}<0.05$ by unpaired, two-tailed Student's t-test comparing the two vaccinated groups.

The study also provides insights for using humanized mouse models for vaccine development and induction of human IgG antibodies in humanized mice.

\section{DISCUSSION}

Humoral immunity is compromised in humanized mice (13). In the present study we reported that CpG-B adjuvant combined with a CD40-targeting vaccination enhanced antigen-specific IgG response. Furthermore, we proved that pre-depletion of human $\mathrm{pDC}$ in vivo abrogated the adjuvant effect of CpG-B. Our results indicated that $\mathrm{CpG}-\mathrm{B}$ and $\mathrm{CD} 40$-targeting vaccination promoted $\mathrm{B}$ cell maturation, triggered human $\mathrm{B}$ cell immunoglobulin classswitch and induced IgG production in a pDC-dependent manner in humanized mice. The findings also shed light on induction of human IgG antibodies in humanized mouse models.

Mice reconstituted with human immune system provides a useful model to study human immunology and vaccinology. Although B cells are developed in humanized mice reconstituted with human HSCs, the cells are immature (13-15, 22-24). The circulating antibody levels, especially IgG levels, are significantly lower compared to adult humans (13). Moreover, the generation of antigen-specific IgG responses in humanized mice, are weak, limiting their application in testing candidate vaccines (13). It was reported that the immunoglobulin gene repertoires of human B cells from humanized mice are similar to those of B cells from human peripheral blood, suggesting that B cells from humanized mice have the genetic potential to produce antibody responses with broad isotype and high affinity (36). Studies have suggested that an absence of human cytokines and the disorganized secondary lymphoid structures might contribute to the defects in B cells (37-39). Signaling TLR results in large amount of cytokines induction in humanized mice. It was reported that pDC-derived IFN- $\alpha$ and IL- 6 were critical for the induction of IgG from human blood mononuclear cells in response to influenza virus (31). pDC preferentially express TLR9 and TLR7 (25). We have previously shown that stimulation of pDC by TLR-9 agonist CpG-B induces IFN- $\alpha$ as well as IL-6 production in vivo in humanized mice (9). In the present study, we found that CpG-B adjuvant combined with CD40-targeting vaccination induced $\mathrm{IgG}^{+} \mathrm{B}$ cells in spleen and PBMCs of humanized mice and induced antigen-specific IgG in serum. This indicated that $\mathrm{CpG}-\mathrm{B}$ and $\mathrm{CD} 40$-targeting vaccination triggers human $\mathrm{B}$ cell immunoglobulin class-switch in humanized mice. We also observed that B cells from mice receiving CpG-B plus CD40-targeting vaccination were more mature in phenotype and express a higher level of the activation marker CD86. The results together suggest that with proper adjuvant, human B cells in this model can produce specific IgG to a vaccine treatment. We also performed CpG-B plus non-CD40 targeting recombinant protein and showed that it did not induce significant IgG response. Thus CD40-targeting, as well as TLR9 activation, was also important for the vaccine to induce classswitch and IgG production. This could be due to CD40 activation of targeted APCs or prolonged antigen-presentation as was observed for T cell epitopes (40).

To prove whether pDCs are important for the IgG induction in vivo, we used a pDC-specific antibody to deplete $\mathrm{pDC}$ before vaccination. Our results showed that depletion of human $\mathrm{pDC}$ in vivo abrogated the $\mathrm{B}$ cell maturation and IgG production in response to $\mathrm{CpG}-\mathrm{B} / \mathrm{CD} 40$-targeting vaccination. The results suggest that human pDCs are essential to mediate the adjuvant effect of CpG-B in vivo. This is consistent with the report that $\mathrm{pDC}$-derived IFN- $\alpha$ and IL-6 were critical for the induction of IgG from human blood mononuclear cells in response to influenza virus (31). We found that pDCs were required for $\mathrm{CpG}-\mathrm{B}$ treatment in humanized mice to induce IFN- $\alpha$ and IL- 6 production. We speculate that IFN- $\alpha$ and IL6 from pDC enhanced B cell maturation and IgG-class switch in response to $\mathrm{CpG}-\mathrm{B}$ plus $\mathrm{CD} 40$-targeting vaccination. It is also 
important to state that there were shortcomings in our study. The antigen we used in this study was not HIV envelop protein so that we were not able to evaluate the neutralization activity of antibody induced by our vaccination stratagem. In another recent study, we proved that TLR-9 agonist plus CD40-targeting HIV envelop vaccination induced HIV-1 envelop-specific IgG with a diversified immunoglobulin repertoire and circulating Env-specific IgGswitched memory human B cells that exhibit clear signs of antigendriven antibody affinity maturation (41).

In summary, we report that CpG-B as an adjuvant promoted human IgG induction through pDC-dependent mechanisms. The proof-of-concept study sheds light on specific induction of human IgG antibodies in humanized mice.

\section{DATA AVAILABILITY STATEMENT}

The original contributions presented in the study are included in the article/supplementary material. Further inquiries can be directed to the corresponding authors.

\section{ETHICS STATEMENT}

The animal study was reviewed and approved by University of North Carolina Institutional Animal Care and Use Committee.

\section{REFERENCES}

1. Lai F, Chen Q. Humanized Mouse Models for the Study of Infection and Pathogenesis of Human Viruses. Viruses (2018) 10(11):643. doi: 10.3390/ v10110643

2. Marsden MD, Zack JA. Humanized Mouse Models for Human Immunodeficiency Virus Infection. Annu Rev Virol (2017) 4:393-412. doi: 10.1146/annurev-virology-101416-041703

3. Shultz LD, Lyons BL, Burzenski LM, Gott B, Chen X, Chaleff S, et al. Human Lymphoid and Myeloid Cell Development in NOD/Ltsz-Scid IL2R Gamma Null Mice Engrafted With Mobilized Human Hemopoietic Stem Cells. J Immunol (2005) 174:6477-89. doi: 10.4049/jimmunol.174.10.6477

4. Zhang L, Kovalev GI, Su L. HIV-1 Infection and Pathogenesis in a Novel Humanized Mouse Model. Blood (2007) 109:2978-81. doi: 10.1182/blood2006-07-033159

5. Ishikawa F, Yasukawa M, Lyons B, Yoshida S, Miyamoto T, Yoshimoto G, et al. Development of Functional Human Blood and Immune Systems in NOD/SCID/IL2 Receptor \{Gamma\} Chain(Null) Mice. Blood (2005) 106:1565-73. doi: 10.1182/blood-2005-02-0516

6. Traggiai E, Chicha L, Mazzucchelli L, Bronz L, Piffaretti JC, Lanzavecchia A, et al. Development of a Human Adaptive Immune System in Cord Blood CellTransplanted Mice. Science (2004) 304:104-7. doi: 10.1126/science.1093933

7. Cheng L, Ma J, Li G, Su L. Humanized Mice Engrafted With Human HSC Only or HSC and Thymus Support Comparable Hiv-1 Replication, Immunopathology, and Responses to ART and Immune Therapy. Front Immunol (2018) 9:817. doi: 10.3389/fimmu.2018.00817

8. Li Y, Masse-Ranson G, Garcia Z, Bruel T, Kok A, Strick-Marchand H, et al. A Human Immune System Mouse Model With Robust Lymph Node Development. Nat Methods (2018) 15:623-30. doi: 10.1038/s41592-0180071-6

9. Cheng L, Zhang Z, Li G, Li F, Wang L, Zhang L, et al. Human Innate Responses and Adjuvant Activity of TLR Ligands in Vivo in Mice Reconstituted With a Human Immune System. Vaccine (2017) 35:6143-53. doi: 10.1016/j.vaccine.2017.09.052

\section{AUTHOR CONTRIBUTIONS}

LC and LS conceived the study and designed the experiments. GL, FL, FY, and CP performed the experiments. LC performed the analyses. LC, JT, and LS interpreted the data, wrote the manuscript, and supervised the study. GZ and SZ provided key reagents. YL helped conceive the overall program for studying anti-CD40 targeting in humanized mice. All authors contributed to the article and approved the submitted version.

\section{FUNDING}

This study was supported in part by NIH grants R01AI136990 (to LS), AI141333 (to JT), AI109784 (to JT and LS), and the Vaccine Research Institute via the ANR-10-LABX-77 grant funded part of this work.

\section{ACKNOWLEDGMENTS}

We thank Liqun Chi for technical support, Ning Jenny Jiang for helping with analyzing B cell maturation, and Qiuchen Zhao and Lulu Shi for editing the manuscript. Lineberger Comprehensive Cancer Center cores, UNC flow cytometry core, DLAM and UNC CFAR for support.

10. Melkus MW, Estes JD, Padgett-Thomas A, Gatlin J, Denton PW, Othieno FA, et al. Humanized Mice Mount Specific Adaptive and Innate Immune Responses to EBV and TSST-1. Nat Med (2006) 12:1316-22. doi: 10.1038/nm1431

11. Meixlsperger S, Leung CS, Ramer PC, Pack M, Vanoaica LD, Breton G, et al. CD141+ Dendritic Cells Produce Prominent Amounts of IFN-Alpha After Dsrna Recognition and Can Be Targeted Via DEC-205 in Humanized Mice. Blood (2013) 121:5034-44. doi: 10.1182/blood-2012-12-473413

12. Cheng L, Wang Q, Li G, Banga R, Ma J, Yu H, et al. TLR3 Agonist and CD40Targeting Vaccination Induces Immune Responses and Reduces HIV-1 Reservoirs. J Clin Invest (2018) 128:4387-96. doi: 10.1172/JCI99005

13. Seung E, Tager AM. Humoral Immunity in Humanized Mice: A Work in Progress. J Infect Dis (2013) 208 Suppl 2:S155-9. doi: 10.1093/infdis/jit448

14. Watanabe Y, Takahashi T, Okajima A, Shiokawa M, Ishii N, Katano I, et al. The Analysis of the Functions of Human B and T Cells in Humanized NOD/ Shi-Scid/Gammac(Null) (NOG) Mice (Hu-HSC NOG Mice). Int Immunol (2009) 21:843-58. doi: 10.1093/intimm/dxp050

15. Biswas S, Chang H, Sarkis PT, Fikrig E, Zhu Q, Marasco WA. Humoral Immune Responses in Humanized BLT Mice Immunized With West Nile Virus and HIV-1 Envelope Proteins are Largely Mediated Via Human CD5+ B Cells. Immunology (2011) 134:419-33. doi: 10.1111/j.1365-2567.2011.03501.x

16. Beutler BA. TLRS and Innate Immunity. Blood (2009) 113:1399-407. doi: 10.1182/blood-2008-07-019307

17. Kawai T, Akira S. The Role of Pattern-Recognition Receptors in Innate Immunity: Update on Toll-Like Receptors. Nat Immunol (2010) 11:373-84. doi: 10.1038/ni.1863

18. O'Neill LA, Bowie AG. Sensing and Signaling in Antiviral Innate Immunity. Curr Biol CB (2010) 20:R328-33. doi: 10.1016/j.cub.2010.01.044

19. Iwasaki A, Medzhitov R. Regulation of Adaptive Immunity by the Innate Immune System. Science (2010) 327:291-5. doi: 10.1126/science.1183021

20. Coffman RL, Sher A, Seder RA. Vaccine Adjuvants: Putting Innate Immunity to Work. Immunity (2010) 33:492-503. doi: 10.1016/j.immuni.2010.10.002

21. Mbow ML, De Gregorio E, Valiante NM, Rappuoli R. New Adjuvants for Human Vaccines. Curr Opin Immunol (2010) 22:411-6. doi: 10.1016/ j.coi.2010.04.004 
22. Chen Q, He F, Kwang J, Chan JK, Chen J. Gm-CSF and IL-4 Stimulate Antibody Responses in Humanized Mice by Promoting T, B, and Dendritic Cell Maturation. J Immunol (2012) 189:5223-9. doi: 10.4049/ jimmunol.1201789

23. Jangalwe S, Shultz LD, Mathew A, Brehm MA. Improved B Cell Development in Humanized NOD-Scid IL2Rgamma(Null) Mice Transgenically Expressing Human Stem Cell Factor, Granulocyte-Macrophage Colony-Stimulating Factor and Interleukin-3. Immun Inflamm Dis (2016) 4:427-40. doi: 10.1002/iid3.124

24. Yu H, Borsotti C, Schickel JN, Zhu S, Strowig T, Eynon EE, et al. A Novel Humanized Mouse Model With Significant Improvement of Class-Switched, Antigen-Specific Antibody Production. Blood (2017) 129:959-69. doi: 10.1182/blood-2016-04-709584

25. Hornung V, Rothenfusser S, Britsch S, Krug A, Jahrsdorfer B, Giese T, et al. Quantitative Expression of Toll-Like Receptor 1-10 Mrna in Cellular Subsets of Human Peripheral Blood Mononuclear Cells and Sensitivity to Cpg Oligodeoxynucleotides. J Immunol (2002) 168:4531-7. doi: 10.4049/ jimmunol.168.9.4531

26. Kadowaki N, Ho S, Antonenko S, Malefyt RW, Kastelein RA, Bazan F, et al. Subsets of Human Dendritic Cell Precursors Express Different Toll-Like Receptors and Respond to Different Microbial Antigens. J Exp Med (2001) 194:863-9. doi: 10.1084/jem.194.6.863

27. Zhang L, Jiang Q, Li G, Jeffrey J, Kovalev GI, Su L. Efficient Infection, Activation, and Impairment of Pdcs in the BM and Peripheral Lymphoid Organs During Early HIV-1 Infection in Humanized Rag2(-)/(-)Gamma C(-)/(-) Mice in Vivo. Blood (2011) 117:6184-92. doi: 10.1182/blood-2011-01-331173

28. Flamar AL, Xue Y, Zurawski SM, Montes M, King B, Sloan L, et al. Targeting Concatenated HIV Antigens to Human CD40 Expands a Broad Repertoire of Multifunctional CD4+ and CD8+ T Cells. AIDS (2013) 27:2041-51. doi: 10.1097/QAD.0b013e3283624305

29. Muramatsu M, Kinoshita K, Fagarasan S, Yamada S, Shinkai Y, Honjo T. Class Switch Recombination and Hypermutation Require Activation-Induced Cytidine Deaminase (AID), a Potential RNA Editing Enzyme. Cell (2000) 102:553-63. doi: 10.1016/S0092-8674(00)00078-7

30. Liu YJ. IPC: Professional Type 1 Interferon-Producing Cells and Plasmacytoid Dendritic Cell Precursors. Annu Rev Immunol (2005) 23:275-306. doi: 10.1146/annurev.immunol.23.021704.115633

31. Jego G, Palucka AK, Blanck JP, Chalouni C, Pascual V, Banchereau J. Plasmacytoid Dendritic Cells Induce Plasma Cell Differentiation Through Type I Interferon and Interleukin 6. Immunity (2003) 19:225-34. doi: 10.1016/S1074-7613(03)00208-5

32. Li G, Cheng M, Nunoya J, Cheng L, Guo H, Yu H, et al. Plasmacytoid Dendritic Cells Suppress HIV-1 Replication But Contribute to HIV-1 Induced Immunopathogenesis in Humanized Mice. PloS Pathog (2014) 10:e1004291. doi: 10.1371/journal.ppat.1004291

33. Grewal IS, Flavell RA. CD40 and CD154 in Cell-Mediated Immunity. Annu Rev Immunol (1998) 16:111-35. doi: 10.1146/annurev.immunol.16.1.111
34. Barr TA, McCormick AL, Carlring J, Heath AW. A Potent Adjuvant Effect of CD40 Antibody Attached to Antigen. Immunology (2003) 109:87-92. doi: 10.1046/j.1365-2567.2003.01634.x

35. Hangalapura BN, Oosterhoff D, Aggarwal S, Wijnands PG, van de Ven R, Santegoets SJ, et al. Selective Transduction of Dendritic Cells in Human Lymph Nodes and Superior Induction of High-Avidity Melanoma-Reactive Cytotoxic T Cells by a CD40-Targeted Adenovirus. J Immunother (2010) 33:706-15. doi: 10.1097/CJI.0b013e3181eccbd4

36. Ippolito GC, Hoi KH, Reddy ST, Carroll SM, Ge X, Rogosch T, et al. Antibody Repertoires in Humanized NOD-Scid-IL2Rgamma(Null) Mice and Human B Cells Reveals Human-Like Diversification and Tolerance Checkpoints in the Mouse. PloS One (2012) 7:e35497. doi: 10.1371/journal.pone.0035497

37. Vuyyuru R, Patton J, Manser T. Human Immune System Mice: Current Potential and Limitations for Translational Research on Human Antibody Responses. Immunol Res (2011) 51:257-66. doi: 10.1007/s12026-011-8243-9

38. Chen Q, Khoury M, Chen J. Expression of Human Cytokines Dramatically Improves Reconstitution of Specific Human-Blood Lineage Cells in Humanized Mice. Proc Natl Acad Sci U.S.A. (2009) 106:21783-8. doi: 10.1073/pnas.0912274106

39. Matsumura T, Kametani Y, Ando K, Hirano Y, Katano I, Ito R, et al. Functional Cd5+ B Cells Develop Predominantly in the Spleen of NOD/ SCID/Gammac(Null) (NOG) Mice Transplanted Either With Human Umbilical Cord Blood, Bone Marrow, or Mobilized Peripheral Blood CD34+ Cells. Exp Hematol (2003) 31:789-97. doi: 10.1016/S0301-472X(03)00193-0

40. Yin W, Gorvel L, Zurawski S, Li D, Ni L, Duluc D, et al. Functional Specialty of CD40 and Dendritic Cell Surface Lectins for Exogenous Antigen Presentation to CD8(+) and CD4(+) T Cells. EBioMedicine (2016) 5:46-58. doi: 10.1016/ j.ebiom.2016.01.029

41. Godot V, Tcherakian C, Gil L, Cervera-Marzal I, Li G, Cheng L, et al. TLR-9 Agonist and CD40-Targeting Vaccination Induces HIV-1 Envelope-Specific B Cells With a Diversified Immunoglobulin Repertoire in Humanized Mice. PloS Pathog (2020) 16:e1009025. doi: 10.1371/journal.ppat.1009025

Conflict of Interest: GZ, SZ, and YL are named inventors on patent applications relevant to $\alpha$ CD40-HIV5pep held by INSERM Transfert.

The remaining authors declare that the research was conducted in the absence of any commercial or financial relationships that could be construed as a potential conflict of interest.

Copyright (C) 2021 Cheng, Li, Pellegry, Yasui, Li, Zurawski, Zurawski, Levy, Ting and $\mathrm{Su}$. This is an open-access article distributed under the terms of the Creative Commons Attribution License (CC BY). The use, distribution or reproduction in other forums is permitted, provided the original author(s) and the copyright owner(s) are credited and that the original publication in this journal is cited, in accordance with accepted academic practice. No use, distribution or reproduction is permitted which does not comply with these terms. 